\title{
Interacting classical dimers on the square lattice
}

\author{
Fabien Alet ${ }^{1}$, Jesper Lykke Jacobsen ${ }^{1,2}$, Grégoire Misguich ${ }^{1}$, Vincent Pasquier ${ }^{1}$, Frédéric Mila ${ }^{3}$, and Matthias Troyer ${ }^{4}$ \\ ${ }^{1}$ Service de Physique Théorique (URA 2306 of CNRS), CEA Saclay, 91191 Gif sur Yvette, France \\ ${ }^{2}$ LPTMS, Université Paris-Sud, Bâtiment 100, 91405 Orsay, France \\ ${ }^{3}$ Institute of Theoretical Physics, Ecole Polytechnique Fédérale de Lausanne, CH-1015 Lausanne, Switzerland and \\ ${ }^{4}$ Theoretische Physik, ETH Zürich, CH-8093 Zürich, Switzerland
}

(Dated: March 20, 2018)

\begin{abstract}
We study a model of close-packed dimers on the square lattice with a nearest neighbor interaction between parallel dimers. This model corresponds to the classical limit of quantum dimer models [D.S. Rokhsar and S.A. Kivelson, Phys. Rev. Lett. 61, 2376 (1988)]. By means of Monte Carlo and Transfer Matrix calculations, we show that this system undergoes a Kosterlitz-Thouless transition separating a low temperature ordered phase where dimers are aligned in columns from a high temperature critical phase with continuously varying exponents. This is understood by constructing the corresponding Coulomb gas, whose coupling constant is computed numerically. We also discuss doped models and implications on the finite-temperature phase diagram of quantum dimer models.
\end{abstract}

The model of lattice coverings by hard-core dimers has a long history [1] in classical statistical physics, culminating in its solution for arbitrary planar graphs [2], the calculation of correlation functions [3] , and its connection to Ising [4] or height models [5, 6]. With the introduction of quantum dimer models (QDM) by Rokhsar and Kivelson [7], and later in connection with resonating valence bond (RVB) and fractionalization physics in 2D [8] and 3D [9], dimer models have regained interest.

As a step towards the understanding of the finitetemperature phase diagram of the QDM, we here study its limit when kinetic terms vanish, which is a classical dimer model with an interaction that tends to align neighboring dimers. The dimer-dimer correlations which are critical in the noninteracting model [3] are found to to remain critical, but with continuously varying exponents, down to a finite temperature where the dimers order through a Kosterlitz-Thouless (KT) transition [10]. Our results are naturally understood in the Coulomb gas approach [1] to the associated height model [5, 6]. Indeed, this interacting dimer model provides one of the simplest realizations (together with the six-vertex and the XY model) of a Coulomb gas (CG) with continuously varying exponents. We further study the effect of doping the model with monomers, and comment on the consequences for the quantum case.

Model - We study a model of interacting dimers on the square lattice, first introduced in the liquid crystal context [12], defined by the partition function

$$
Z=\sum_{\{c\}} \exp \left[-\frac{v}{T}\left(N^{c}(\mathbf{\Xi})+N^{c}(\mathbf{I I})\right)\right]
$$

where $T$ is the temperature, the sum runs over all dimer coverings $\{c\}$, and $N^{c}(\mathbf{=})+N^{c}(\mathbf{I I})$ is the number of plaquettes with parallel dimers in configuration $c$. We mainly consider the case $v=-1$, with aligning interactions between dimers. Note that the model Eq. (11) corresponds to the diagonal part of the QDM Hamiltonian [].

At $T=\infty$, Eq. (11) describes the classical dimer cov-

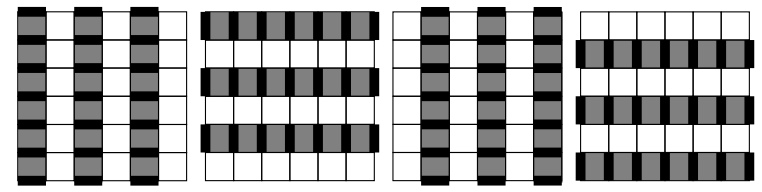

FIG. 1: The four possible columnar ground states. To each plaquette with a pair of parallel dimers we assign an energy of $+v$ (shaded plaquettes in the figure).

ering problem, and thus displays critical correlations [3]. At $T=0$, the dimers order in columns and the groundstate is four-fold degenerate, as shown in Fig. 11 This columnar phase breaks translational and rotational symmetry.

We study Eq. (1) with Transfer Matrix (TM) techniques and with a Monte Carlo (MC) directed loop algorithm [13]. The $\mathrm{MC}$ simulations are made on $N=L \times L$ lattices with periodic boundary conditions (PBC).

Transition to the columnar phase - Previous simulations of the QDM on the square lattice found the existence of a plaquette phase (which breaks translational but not $\frac{\pi}{2}$-rotational symmetry) [14]. In the MC simulations of the classical model Eq. (11), we also find important plaquette correlations on finite samples, which nevertheless vanish in the thermodynamic limit 15]. However, they affect the finite-size scaling analysis of the order parameter used in Ref. 16. For this reason we resort to quantities insensitive to plaquette correlations to detect the entrance into the columnar phase. Following Ref. 14, we define the dimer rotational symmetry breaking (DSB) and pair rotational symmetry breaking (PSB) order parameters as:

$$
\begin{aligned}
\mathrm{DSB} & =N^{-1}\left|N^{c}(\mathbf{-})-N^{c}(\mathbf{I})\right| \\
\mathrm{PSB} & =N^{-1}\left|N^{c}(\mathbf{(})-N^{c}(\mathbf{I} \mathbf{I})\right|,
\end{aligned}
$$

with $N^{c}(\mathbf{-})\left(N^{c}(\mathbf{I})\right)$ the number of horizontal (vertical) dimers in the configuration $c$. Both quantities show longrange order in a columnar phase, but vanish in a plaque- 


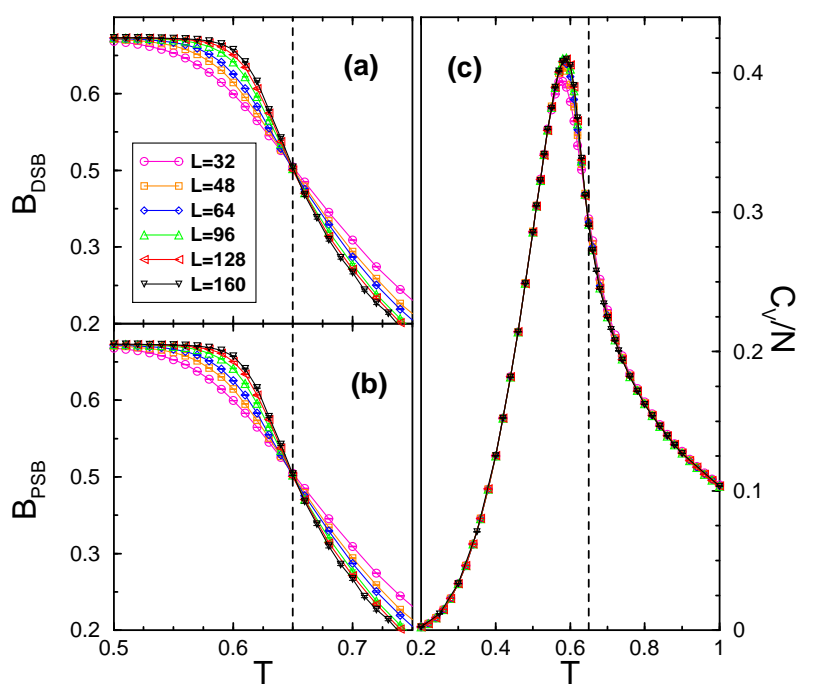

FIG. 2: (Color online) Dimer symmetry breaking cumulant (a), Pair symmetry breaking cumulant (b), and Specific heat per site (c) versus temperature for different system sizes. The dashed lines indicate the estimate $T_{c}=0.65(1)$.

tte phase. To locate the transition with high precision, we study Binder cumulants [17], defined as $B_{\mathrm{DSB}}=1-$ $\left\langle\mathrm{DSB}^{4}\right\rangle /\left(3\left\langle\mathrm{DSB}^{2}\right\rangle^{2}\right)$ and $B_{\mathrm{PSB}}=1-\left\langle\mathrm{PSB}^{4}\right\rangle /\left(3\left\langle\mathrm{PSB}^{2}\right\rangle^{2}\right)$; here $\langle\cdots\rangle$ denotes the ensemble average. In the thermodynamic limit, these cumulants saturate to $2 / 3$ in a longrange ordered phase, and vanish in a disordered phase. For both cumulants, the curves for different $L$ all cross at a unique temperature $T$ (see Figs. 2(a) and (b)), which we identify as the transition temperature $T_{\mathrm{c}}=0.65(1)$ to the columnar phase.

The specific heat per site $C_{v} / N$ displays a peak, which does not diverge in the thermodynamic limit [Fig. 2[(c)]. This points towards a second order transition with a critical exponent $\alpha \leq 0$, or a KT transition. The peak of $C_{v} / N$ is located slightly below the value of $T_{c}$ determined by the cumulants. A featureless specific heat at $T_{c}$ is a strong indication of a KT transition.

High temperature phase - For $T>T_{c}$ we calculate the dimer-dimer correlation function

$$
G(\mathbf{x})=\left\langle n_{\mathbf{m}}(\mathbf{r}) n_{\mathbf{-}}(\mathbf{r}+\mathbf{x})\right\rangle-1 / 16
$$

where $n_{\mathbf{-}}(\mathbf{r})=1$ for a horizontal dimer at site $\mathbf{r}$, and 0 otherwise. The constant $1 / 16$ stands for the dimer density squared. Taking $\mathbf{x}=(x, 0)$ we find that $G(x)$ is staggered with $x$ at any $T>T_{c}$. We also calculate the monomer-monomer correlation function $M(\mathbf{x})$, proportional to the number of configurations with two test monomers separated by $\mathbf{x}$, and normalized by $M(1)=$ $1[3,13,18 . M(\mathbf{x})$ can be computed in the loop building process of the MC simulation [13]. For a bipartite system, $M(\mathbf{x})=0$ for monomers on the same sublattice.

Anticipating the results, we look for algebraic decay of these correlators for $T>T_{c}$ and define exponents $\alpha_{d}$ and

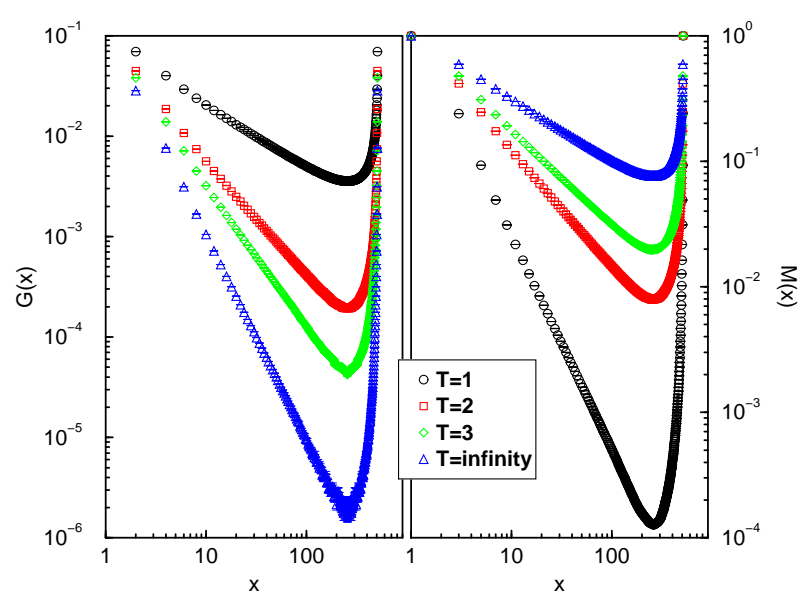

FIG. 3: (Color online) Dimer-Dimer $G(x)$ (left) and Monomer-Monomer $M(x)$ (right) correlation functions versus distance $x$ in a $\log$-log scale for various temperatures.

$\alpha_{m}$ by $G(x) \sim(-)^{x} x^{-\alpha_{d}}$ and $M(x) \sim x^{-\alpha_{m}}$ for large $x$. At $T=\infty$, we have the exact results $\alpha_{d}=2$ and $\alpha_{m}=$ $1 / 2$ [3]. In Fig. 3] we show $G(x)$ and $M(x)$ calculated on an $L=512$ sample for various temperatures $T>$ $T_{\mathrm{c}}$. Both correlators indeed show power-law decay for all $T>T_{\mathrm{c}}$, and the exponents $\alpha_{d}$ and $\alpha_{m}$ appear to vary continuously with $T$. The power-law decay is eventually truncated around $L / 2$ due to the PBC, making a high precision fit for $\alpha_{d}(T)$ and $\alpha_{m}(T)$ difficult.

We therefore turn to TM calculations, which are performed on semi-infinite cylinders of even circumference $L$. By convention, the space coordinate $x$ is horizontal and time $t$ runs upwards. Placing arrows on all lattice edges, such that even (odd) sites are sources (sinks) of four arrows, we define a (staggered) reference configuration $c_{0}$ by placing dimers on up-pointing arrows only. Superposing a given configuration $c$ with $c_{0}$ gives a transition graph with a conserved number $W_{x}$ of (oriented) time-like strings. More precisely, $W_{x}=\sum_{x}\left(n_{\mathbf{i}}^{\uparrow}(x, t)-\right.$ $\left.n_{\mathbf{1}}^{\downarrow}(x, t)\right) \in\left\{-\frac{L}{2}, \ldots, \frac{L}{2}\right\}$ is independent of $t$ and labels the blocks of the TM. The block $W_{x} \neq 0$ corresponds to a defect of $\left|W_{x}\right|$ monomers on the same sublattice at $t=-\infty$, and $\left|W_{x}\right|$ monomers on the opposite sublattice at $t=\infty$. This can be seen by taking a $W_{x}=0$ configuration and shifting its dimers along some time-like string: this changes $W_{x}$ by \pm 1 and introduces a monomer at either end of the string.

Ordering the TM eigenvalues as $\lambda_{1}^{W_{x}} \geq \lambda_{2}^{W_{x}} \geq \ldots$, we expect $\lambda_{1}^{0}$ (no monomers) to dominate all other eigenvalues. Using standard predictions of conformal field theory [19], the $L$-dependence of $\lambda_{1}^{0}$ gives the central charge $c$; the ratio $\lambda_{1}^{0} / \lambda_{2}^{0}$ determines $\alpha_{d}$ and $\lambda_{1}^{0} / \lambda_{1}^{1}$ gives $\alpha_{m}$. Note that the eigenvectors corresponding to $\lambda_{1}^{0}$ (resp. $\lambda_{2}^{0}$ ) are even (resp. odd) under the shift $x \rightarrow x+1$.

To account for the interactions in Eq. (II), the TM states must encode vertical and horizontal dimer occu- 


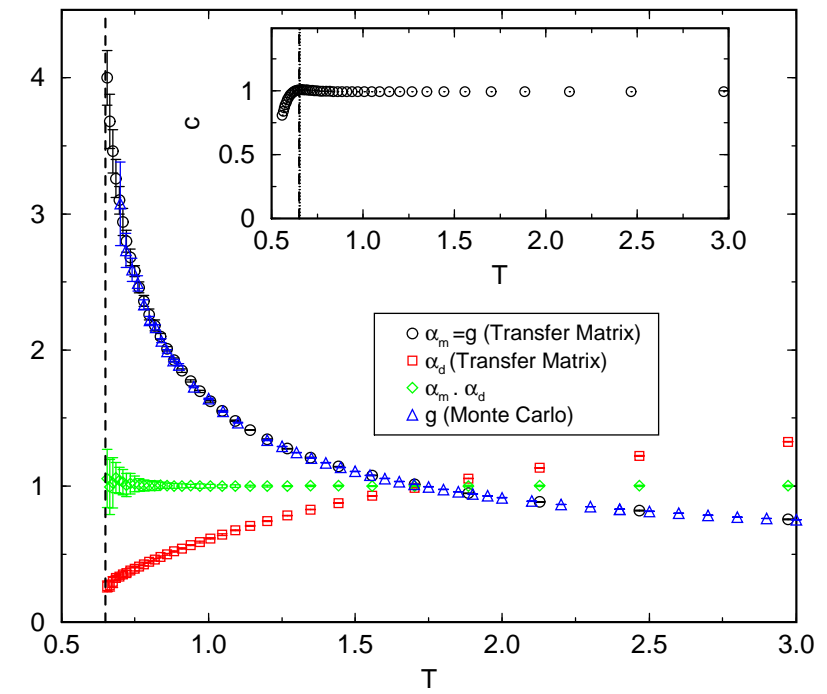

FIG. 4: (Color online) Dimer-dimer $\alpha_{d}$ and monomermonomer $\alpha_{m}$ exponents (and their product) obtained by TM calculations, and coupling constant $g$ of the CG as obtained by $\mathrm{MC}$, all versus temperature $T$. Inset: central charge $c$ versus $T$. The dashed lines denote the MC estimate of $T_{c}$.

pancies within a row [15]. We use sparse-matrix factorization, and the state space is built from a reference state in the given $W_{x}$-sector by means of hashing techniques. Computations were performed up to $L=18$, with 1728292 states in the $W_{x}=0$ sector.

In Fig. 4. we show $\alpha_{m}$ and $\alpha_{d}$ as functions of temperature (error bars are determined from the finite size scaling analysis). $\alpha_{m}$ increases from $1 / 2$ at $T=\infty$ to $4.0(2)$ at the transition temperature $T_{\mathrm{c}}$, and then diverges below

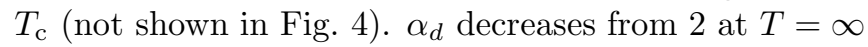
to $0.26(4)$ at $T_{\mathrm{c}}$, and vanishes below (not shown). We also plot in Fig. 固 the product $\alpha_{m} \cdot \alpha_{d}$, which equals 1 within error bars for any $T \geq T_{\mathrm{c}}$. The continuous variation of both $\alpha_{m}$ and $\alpha_{d}$ as functions of $T$ indicates criticality for $T_{\mathrm{c}} \leq T \leq \infty$. This is corroborated by the central charge $c$ as a function of $T$ (see inset of Fig. (4), which is 1.00(1) in the critical phase and drops abruptly below $T_{\mathrm{c}} . T_{\mathrm{c}}$ can be estimated from the TM by $\alpha_{m}\left(T_{\mathrm{c}}\right)=4$, the jump in $c$, or $\alpha_{d}\left(T_{\mathrm{c}}\right)=1 / 4$. The exact values quoted are accounted for by the CG formalism discussed below. The first determination is the most precise and gives $T_{\mathrm{c}}=0.65(1)$, in full agreement with the MC results.

Coulomb gas representation - We now account for the above findings (KT transition, critical phase with continuously varying exponents) by dressing the model's CG representation [11]. Close-packed dimers on the square lattice admit a height representation [5, [6], where a height $z$ is assigned (up to an overall constant) to each plaquette in the following way: going counterclockwise around a site on the even sublattice, $z$ changes by $+3 / 4$ when crossing a dimer and by $-1 / 4$ across an empty bond. In these units, a monomer corresponds to a dislocation of
1 in the height. To describe the long-wavelength physics of our model, we follow Ref. $\underline{6}$ and coarse-grain the local height variable $z$ to a continuum field $h(\mathbf{r})$, for which we postulate the following action:

$$
S=\int d \mathbf{r}\left[\pi g|\nabla h(\mathbf{r})|^{2}+V \cos (2 \pi p h(\mathbf{r}))\right]
$$

The first term involving the CG coupling $g[20]$ accounts for the dimer entropy associated with a given coarsegrained height $h(\mathbf{r})$; it dominates in the high- $T$ rough phase. The second term is a locking potential of strength $V>0$ (the actual value of $V$ is immaterial) that favors dimer ordering in one of the $p=4$ "ideal" states [6] (see Fig. (1) satisfying $h(\mathbf{r})=1 / 8,3 / 8,5 / 8$ and $7 / 8(\bmod 1)$; it dominates in the low- $T$ flat phase. When $T \rightarrow T_{c}$, the locking potential becomes irrelevant and the height model undergoes a roughening transition.

At $T>T_{c}$ the height field is thus free and described by a Coulomb gas with electric charges $e$ only [1]. Dual, magnetic charges $m$ correspond to height dislocations, which can be inserted "by hand" through monomers or appropriate boundary conditions (cf. the above discussion of the TM). Units are such that $e$ and $m$ are integers. The exponent of an electromagnetic operator of charge $(e, m)$ reads $\alpha(e, m)=g^{-1} e^{2}+g m^{2}$ [1]. We identify the leading dimer and monomer correlations by $\alpha_{d}=\alpha(1,0)$ and $\alpha_{m}=\alpha(0,1)$. The exact results of Ref. 3 then fix $g=1 / 2$ at $T=\infty[\underline{6}]$; in general $g=g(T)$ must depend on $T$ to account for the continuously varying exponents.

More generally, electric charges define the vertex operators $V_{e}(\mathbf{r})=\exp (2 \pi i e h(\mathbf{r}))$ : appearing in the Fourier expansion of any operator which is periodic in $h(\mathbf{r})$. Note that $V_{1}$ appears as a continuum limit contribution to the dimer operator 21]. The locking potential corresponds to $V_{4}$ whose marginality determines $g\left(T_{\mathrm{c}}\right)=4$.

To check these CG predictions, we calculate $g(T)$ from

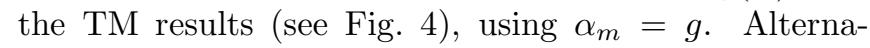
tively, $g(T)$ is accessible from MC calculations of the fluctuations of the winding number $\left\langle W^{2}\right\rangle=\frac{1}{2}\left\langle W_{x}^{2}+W_{y}^{2}\right\rangle$. The winding number $W_{x}$ (resp. $\left.W_{y}\right)$ is the local height difference accumulated by a path winding around the torus with $\mathrm{PBC}$ in the $x$ (resp. $y$ ) direction. $\left\langle W^{2}\right\rangle$ is simply related to $g$ in the free-field point of view [22]. By separating the average tilt from the spatial fluctuations of the height, the partition function is written as a sum over $W_{x}$ and $W_{y}$. The associated Boltzmann weight is given by the action of Eq. (4) evaluated with its classical solution $(\Delta h(\mathbf{r})=0)$ given by a linear height $h(x, y)=\frac{x}{L_{x}} W_{x}+\frac{y}{L_{y}} W_{y}($ and $V=0)$. Then

$$
\left\langle W^{2}\right\rangle=\sum_{n \in \mathbb{Z}} n^{2} \mathrm{e}^{-g \pi n^{2}} / \sum_{n \in \mathbb{Z}} \mathrm{e}^{-g \pi n^{2}} .
$$

Note that at $g=1 / 2$, this is a non-trivial statement about the Pfaffian formulation of the partition function 22. In Fig. 4] we show the MC results for $g$ obtained 
from $\left\langle W^{2}\right\rangle$ and Eq. (5), which match with high precision the TM evaluation. This validates the CG scenario for the transition in our model.

The CG description also holds for $v=+1$, i.e., when dimer alignment is penalized. In this case, the CG coupling decreases from $g=1 / 2$ at $T=\infty$ to $g=0$ at $T=T_{t}$. As $T \rightarrow T_{t}$ the height model becomes unstable and undergoes a tilting transition. When $T<T_{t}$ the winding $\left\langle W^{2}\right\rangle$ becomes extensive. Analysis of the $L$-dependence of the effective central charge gives the estimate $T_{t}=0.449(1)$. More details will appear in Ref. 15.

Doping with monomers - If we allow monomers in the model, the height mapping is lost but the CG description stays valid. For $T>T_{c}$ introducing monomers is a relevant perturbation $\left(\alpha_{m}<4\right)$ and destroys the critical phase, but it becomes marginal at $T_{c}$. Denoting the weight of a monomer by $\mathrm{e}^{\mu / T}$ and the chemical potential by $\mu$, we therefore expect a further critical line $\mu(T)$ to emanate from $\mu\left(T_{c}\right)=-\infty$ and extend towards decreasing $T$. Physically this line separates a (weakly) doped dimer crystal from a liquid of dimers and monomers. The ground-state has only monomers for $\mu /|v|>1 / 2$ but is a 4 -fold degenerate monomer-free crystal when $\mu /|v|<1 / 2$. The transition is thus first order at $T=0$ and presumably remains so for small $T$ as both phases have gapped elementary excitations at $\mu /|v|=1 / 2$. However, the transition at low monomer doping is second order, so it must cease to be continuous at some finite $\left(T_{*}, \mu_{*}\right)$. Using a modified TM we estimate $T_{*}=0.39(4)$ and $\mu_{*} /|v|=0.25(10)$. Along the line, for $T_{*}<T<T_{c}$, the TM results are consistent with $c=1$ and a $T$-independent exponent $\alpha_{d}=1 / 4$. Less relevant operators however have $T$-dependent exponents.

Discussion and conclusion - In conclusion, we have studied a model of dimers on the square lattice with an interaction that favors dimer alignment. We find that this model undergoes a KT transition separating a high- $T$ critical phase with continuously varying exponents from a low- $T$ crystalline phase. The transition is understood as the roughening transition in the height representation or equivalently as a proliferation of $e=4$ electric charges in the CG framework. Our model captures naturally all the contents of the CG: electric (dimers) and magnetic (monomers) charges, and a $T$-dependent coupling constant $g$ driving the transition. Concerning quantum systems, the rough phase should survive in a large part of the finite-temperature phase diagram of the QDM (above the melting temperatures of the ordered phases) and especially so in the vicinity of the Rokhsar-Kivelson point [7]. We thus expect continuously varying exponents parametrized by a CG coupling constant at sufficiently high $T$. Our results also indicate that doping the QDM would immediately destroy this critical phase. Detailed finite-temperature properties of the QDM yet remain to be explored. It would be interesting as well to study quantum Hamiltonians with ground state corresponding to Eq. (11) 23].

Acknowledgments - We thank C. L. Henley, W. Krauth, R. Moessner and A. Ralko for fruitful discussions. The MC calculations were performed on the Gallega cluster at SPhT using the ALPS libraries 24].

[1] J. K. Roberts, Proc. Roy. Soc. (London) A 152, 464 (1935); R. H. Fowler and G.S. Rushbrooke, Trans. Faraday. Soc. 33, 1272 (1937).

[2] P.W. Kasteleyn, Physica 27, 1209 (1961); H.N.V. Temperley and M.E. Fisher, Phil. Mag. 6, 1061 (1961); M. E. Fisher, Phys. Rev. 124, 1664 (1961).

[3] M. E. Fisher and J. Stephenson, Phys. Rev. 132, 1411 (1963).

[4] M. E. Fisher, J. Math. Phys. 7, 1776 (1966).

[5] H. W. J. Blöte and H. J. Hilhorst, J. Phys. A 15, L631 (1982); B. Nienhuis, H. J. Hilhorst and H. W. J. Blöte, ibid. 17, 3559, (1984).

[6] J. Kondev and C. L. Henley, Phys. Rev. B 52, 6628 (1995); Nucl. Phys. B 464, 540 (1996); C. Zeng and C. L. Henley, Phys. Rev. B 55, 14935 (1997); R. Raghavan, C. L. Henley and S. L. Arouh, J. Stat. Phys. 86, 517 (1997).

[7] D.S. Rokhsar and S.A. Kivelson, Phys. Rev. Lett. 61, 2376 (1988).

[8] R. Moessner and S. L. Sondhi, Phys. Rev. Lett. 86, 1881 (2001); G. Misguich, D. Serban and V. Pasquier, ibid. 89, 137202 (2002); R. Moessner, S. L. Sondhi and E. Fradkin, Phys. Rev. B 65, 024504 (2002); D. A. Ivanov, ibid. 70, 094430 (2004).

[9] R. Moessner and S.L. Sondhi, Phys. Rev. B 68, 184512 (2003).

[10] J.M. Kosterlitz and D.J. Thouless, J. Phys. C 6, 1181 (1973); V.L. Berezinskii, Sov. Phys. JETP 32, 493 (1971)

[11] B. Nienhuis, in Phase transitions and Critical Phenomena, edited by C. Domb and J. L. Lebowitz (Academic, London, 1987), Vol. 11.

[12] O.J. Hellmann and E. Præstgaard, Chem. Phys. 24, 119 (1977); D. Poland and P.K. Swaminathan, J. Chem. Phys. 71, 1926 (1979).

[13] A. Sandvik, E-print cond-mat/0312097

[14] P. W. Leung, K. C. Chiu and K. J. Runge, Phys. Rev. B 54, 12938 (1996).

[15] F. Alet et al., in preparation.

[16] S. Sachdev, Phys. Rev. B 40, 5204 (1989).

[17] K. Binder, Z. Phys. B 43, 119 (1981).

[18] W. Krauth and R. Moessner, Phys. Rev. B 67, 064503 (2003).

[19] J.L. Cardy, J. Phys. A 17, L385 (1984); H.W.J. Blöte, J.L. Cardy, and M.P. Nightingale, Phys. Rev. Lett. 56, 742 (1986); I. Affleck, ibid. 56, 746 (1986).

[20] We use here the notations of Ref. 11. $g$ is related to the stiffness constant $K$ used in Refs. 6 by $g=8 K / \pi$.

[21] E. Fradkin et al., Phys. Rev. B 69, 224415 (2004).

[22] S. K. Yang, Nucl. Phys. B 285, 183 (1987); P. Di Francesco, H. Saleur and J.-B. Zuber, J. Stat. Phys 49, 57 (1987).

[23] C.L. Henley, J. Phys. C 16, S891 (2004); C. Castelnovo et al., E-print cond-mat/0502068

[24] F. Alet et al., E-print cond-mat/0410407 M. Troyer, B. Ammon and E. Heeb, Lect. Notes Comput. Sci., 1505, 191 (1998). See alo http://alps.comp-phys.org 